the non-compliance was caused by confusion over the discrepancy between blood sugar levels measured on bedside and blood gas machines. This led to a guideline amendment that blood sugars less than $2.5 \mathrm{mmol} / \mathrm{L}$ should now be confirmed with capillary gas sample. A further staff survey showed improvement in guideline familiarity.

Lessons learnt Success of this quality improvement project relied on information from all members of the multidisciplinary team. Their survey responses helped us identify interventions required. We feel the presence of human champions to continue reinforcing the message has helped us maintain good practice.

Message for others Our main message is the value of human factors to maintain good practice and involving all members of multidisciplinary team in quality improvement work. Our project has also shown the importance of addressing problems at all levels.

\section{G514 SMALL, SMART AND SUSTAINABLE; CHANGES WITHIN A ZAMBIAN CHILDREN'S WARD}

S Alexander. Medical Paediatrics, Royal Hospital for Sick Children, Edinburgh, UK

\subsection{6/archdischild-2015-308599.467}

Context This work was performed at St Francis Hospital, Zambia between February and July 2010. St Francis is a 340 bed hospital in rural Zambia. The number of Medical Staff at the hospital varies from 4 to 20 relying predominantly on volunteers. Zambian Clinical Officers perform a role similar to an Advanced Nurse Practitioner and there are many Midwives, Nurses and students.

The Paediatric ward has an Intensive Care (IC) area with eight beds, two oxygen concentrators, one suction machine and no infusion pumps. There are 28 malnutrition beds, 68 general beds and 18 cots. Inpatient numbers range from 3 to 300 depending on seasonal variation.

Problem My intention was to investigate antibiotic sensitivity to respiratory pathogens. In reality, performing such a study in five months is impossible. You have to manage the acute workload, overcome cultural and language barriers whilst gaining the trust of the staff. My agenda quickly changed.

The admission of a child would typically entail a journey on foot to the hospital, a queue at Outpatients, an assessment by a Clinical Officer then a queue for a cannula. Very sick children would be placed in an IC bed meaning they were reviewed twice a day. New admissions would be seen by a doctor the following morning. No child would have observations recorded.

Intervention Firstly we addressed the problem of children arriving at the ward in a hypoglycaemic coma. We made glucose water and cups available to anyone waiting in the queue at outpatients. Secondly, when possible we assigned a Clinical Officer to paediatric triage so that children were a priority. We rearranged the Intensive Care area to create more beds and introduced an incubator for the acutely unwell babies with malnutrition. We created a portable emergency trolley from a disused wooden cart.

The biggest change was a medical presence on the ward from 08.00-18.00. A daily ward round was conducted and the afternoons spent reviewing those in the IC area and new admissions. The aim was to ensure patients had a nasogastric tube sited with regular glucose water and other appropriate medication. A national blood shortage meant provisions were made for parent to child or staff to child transfusions. We gave tutorials on recognising the acutely unwell child and Neonatal teaching.

\begin{tabular}{|l|l|l|l|l|l|l|l|}
\hline $\begin{array}{l}\text { Abstract G514 Table } 1 \\
2010\end{array}$ & \multicolumn{5}{|c|}{ Paedatric mortality rates January - June } \\
\hline Month & Jan & Feb & March & April & May & June \\
\hline Admission & 850 & 768 & 828 & 808 & 1016 & 530 \\
\hline Deaths & 91 & 79 & 73 & 68 & 80 & 42 \\
\hline Percentage & $10.7 \%$ & $10.3 \%$ & $8.8 \%$ & $8.4 \%$ & $7.8 \%$ & $7.2 \%$ \\
\hline
\end{tabular}

Results Results were assessed by examining mortality statistics over the 5 month period as can be seen in Table 1 . We presented these results to the hospital and the statistics were used in a Government bid for funding for a permanent Paediatric Clinical Officer.

Discussion Projects attempted in developing countries require time and planning. Advance contact with the hospital and knowledge of resources is essential but can be difficult with poor internet access and work pressures. Current Specialist Training does not lend itself easily to significant time out of programme. However in a short period of time, by addressing small problems and setting achievable goals, quality improvement measures can be made and have a lasting effect.

\section{G515 PEST - PRESCRIBING ERROR SURVEILLANCE TEAM}

D James, C Bevan, M Rahman, S Chakraborty. Royal Alexandra Childrens Hospital, Brighton and Sussex Medical School, Brighton, UK

\subsection{6/archdischild-2015-308599.468}

Context This quality improvement project was carried out at a large general paediatric hospital. The project was performed on our medical and surgical inpatient wards, involving all prescribing healthcare professionals.

Problem There had been numerous minor and moderate prescribing errors identified via DATIX and CPSQ meetings, the majority not affecting patient care and were identified prior to administration of medication, thus avoiding serious incidents. However, they caused delays in treatment and sub-optimal, timely patient care. This inefficient practice wastes time and resources of nurses, doctors and pharmacists in identification of errors and re-prescribing of medication.

Assessment of problem and analysis of its causes This problem had already been identified at CPSQ meetings and the medication errors were ongoing despite improvement in prescribing training at staffing induction.

A multidisciplinary meeting involving doctors, senior nursing clinicians and the pharmacy lead was held to identify common errors. The prescribing errors were primarily illegible handwriting, inaccurate history taking, confusion with drug names, inappropriate use of decimal points, incorrect completion of drug charts and use of verbal orders and abbreviations. Clinicians were often unaware of errors, with colleagues amending drug charts, with no direct feedback to the person making the error.

Intervention Our quality improvement intervention was to implement a "zero tolerance policy" of prescribing errors, such that no medication would be given until correctly prescribed. No verbal orders were accepted. All errors once identified were photographed by paediatric pharmacists. All photographic 